\title{
Analysis of People Trajectories with Ubiquitous Sensors in a Science Museum
}

\author{
Takayuki Kanda, Masahiro Shiomi, Laurent Perrin, Tatsuya Nomura, Hiroshi Ishiguro, and Norihiro Hagita
}

\begin{abstract}
This paper reports a study that estimated visitor positions, visiting patterns, and inter-human relationships at a science museum using information from RFID readers. In the science museum, we exhibited humanoid robots. Visitors were invited to wear RFID tags to interact with the robots. Visitor behavior was simultaneously observed using 20 RFID readers, distributed throughout the entire floor, that roughly measured the distances of nearby tags. We integrated the outputs from all RFID readers to estimate visitor trajectories that were used to analyze three perspectives: space, visiting patterns, and relationships. Regarding space, we identified crowded and uncrowded areas. We found several typical visiting patterns, such as visited at every exhibit and directly going to robot area. We also identified atypical visiting behavior. Regarding relationships, for example, we estimated $68 \%$ coverage of group-member relationships with $91 \%$ reliability.
\end{abstract}

\section{INTRODUCTION}

$\mathrm{R}$ ECENT developments in ubiquitous computing and robotics enable us to integrate these fields to produce an idealized human-computer interface called the "network robot system." In this context humanoid robots serve an informative role with support from ubiquitous sensors. There are several previous research works in this area, such as projects that used RFID tags to identify interacting children to further promote interactions with robots $[1,2]$. However, what a single robot can recognize is limited, even if many RFID tags are distributed throughout the environment on the people within it. A strong need exists to acquire a greater understanding of human activities through the use of ubiquitous sensors.

Several research efforts have also been conducted into recognizing human positions and trajectories using ubiquitous sensors [3-6]. Ubiquitous computing technology is gradually being used to analyze people's activities. For example, Eagle and Pentland developed a Bluetooth-based device attached to a mobile phone that enables us to recognize such routine activities as being at home, in an office, or

Manuscript received September 15, 2006. This research was supported by Special Coordination Funds for Promoting Science and Technology of the Ministry of Education, Culture, Sports, Science and Technology (MEXT), Japan.

All authors are with the ATR Intelligent Robotics and Communication Laboratories, 2-2-2 Hikaridai, Keihanna Science City, Kyoto, Japan, e-mail: kanda@atr.jp). In addition, M. Shiomi and H. Ishiguro are with Osaka University, Suita City, Osaka, Japan elsewhere [7]. Liao et al. also used locations obtained via GPS with a relational Markov model to discriminate location-based activities [8]. Choudhury and Pentland developed a wearable device called a "sociometer" to visualize people's peer relationships based on vocal communication activity [9].

Scant previous research, however, has revealed to what extent we can identify people's behavior by studying their trajectories. Although Sparacino classified visitors into three pre-defined types based on trajectories obtained through wearable sensors [10], the user types were defined in advance, such as 'busy,' 'selective,' and 'greedy', and have been developed in museological study with time-consuming observation by researchers [11].

We are particularly interested in exploring in an exhibition environment such as a museum, where many people remain in an environment over an extended period of time and walk and look around. In such an environment, we believe that people's trajectories provide information that can be used to promote activities in the environment. For example, we expect that people will move around to see what they are interested in; thus, the amount of time a person remains at a certain position may reflect his/her interest in a particular exhibit.

This paper addresses to what extent we can estimate people's activities in a museum space from their trajectories observed by ubiquitous sensors. We conducted a 25-day field trial in a science museum involving humanoid robots, whose presence offered considerable temptation to visitors to participate in the field trial. During the 25 days, 8,091 visitors participated in the field trial by wearing RFID tags and interacting with the robots. We chose RFID as a positioning device since RFID tags are easy to carry and we can observe a number of tags at the same time. Analysis of participant trajectories revealed conclusions from three different perspectives: space, visiting patterns, and relationships.

\section{II.DATA COLLECTION}

\section{A. Field trial at a science museum}

The study was conducted in conjunction with a field trial with humanoid robots at the Osaka Science Museum in Japan. This subsection briefly explains the field trial (See [2] for details). Figure 1 shows a floor plan of the museum space that was used. It is approximately 84 x $42 \mathrm{~m}$. Visitors entered 


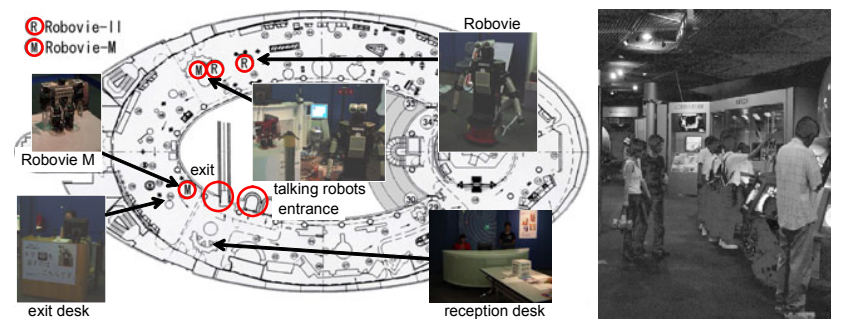

Figure 1: Fourth floor of Osaka science museum
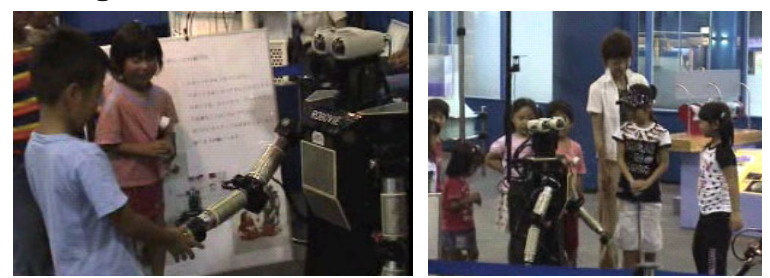

Figure 2: Interaction scene with a humanoid robot, Robovie-II

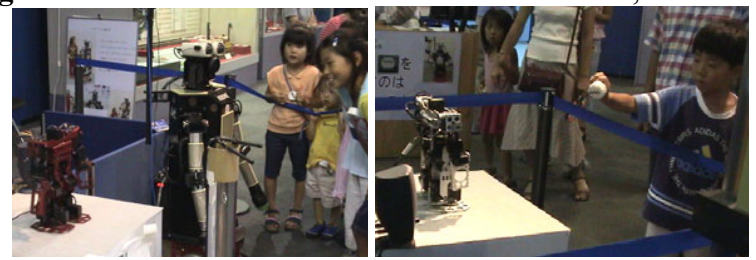

Figure 3: Interaction scene with other humanoid robots

using the elevator shown at the bottom left of the plan, and at the reception desk they were invited to participate in the field trial. Participants were asked to wear RFID tags. If a visitor decided to participate, he/she completed a registration form whose information was stored in a database server. Visitors were free to look around the museum as they wished. For this purpose, 400 RFID tags were prepared; at most 172 RFID tags were simultaneously active (worn by visitors).

Four humanoid robots, Robovie-II and Robovie-M, were placed in the environment at positions $\mathrm{R}$ and $\mathrm{M}$ shown in Figure 1. The robots had three roles. One Robovie-II guided people to museum exhibits while it is moving around, where its position is revised by infrared sensor attached to the robot and ceiling. This robot can engage in such behavior as handshaking, hugging, and the rock-paper-scissors game, as shown in the left of Figure 2. It can also guide people to four kinds of exhibits, as shown in Figure 2 on the right. When bringing visitors to the telescope, the robot says, "I am taking you to an exhibit, please follow me!" It then approaches the telescope and explains it.

Robovie-II is equipped with an RFID reader (the detail of which are explained in section 2.2). When it detects an RFID tag worn by a participant, it uses the registered information stored in the database to promote interaction with visitors, such as greeting them by name. Robovie-II also used the amount of time that visitors spent near particular exhibits to judge whether visitors had used the exhibits. For example, when an RFID-tagged visitor had lingered around the "magnetic power" exhibit more than a predefined time, the system
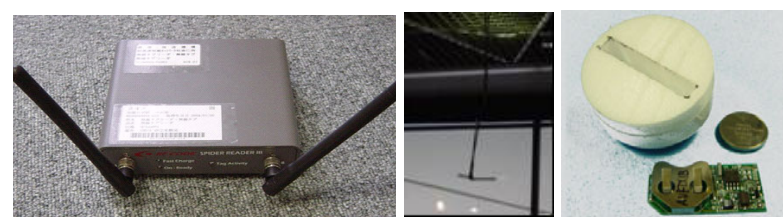

Figure 4: RFID reader, antenna attached to ceiling, and tag

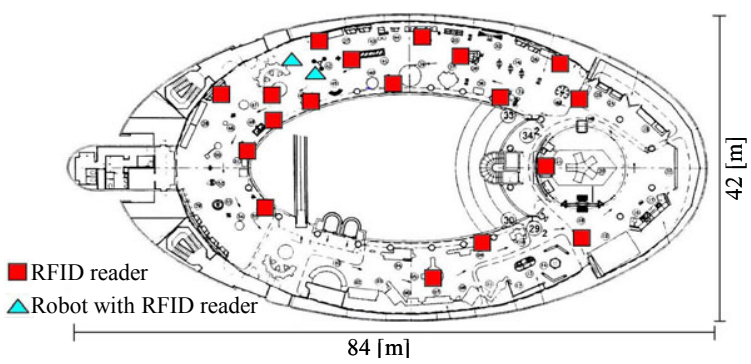

Figure 5: Placement of RFID tags

would assume that the visitor had tried it. The robot might say, "Yamada-san, thank you for trying 'magnetic power.' What did you think of it?" Participants enjoyed such interaction with robots, particularly when personally greeted by name. Visitors not wearing RFID tags were also free to interact with the robots outside of experiencing such greeting behavior.

The other Robovie-II and one of the Robovie-M explained about exhibits behaved as if they were talking (Figure 3, left). The remaining Robovie-M did not provide guidance but instead interacted with visitors by using their names from visitor tags and registered information, saying "good-bye," and asking visitors to return their tags (Figure 3, right). The other Robovie-II was also equipped with an RFID reader and the remaining Robovie-M utilized a nearby RFID reader embedded in the environment. In the figure, the boy is showing his RFID tag to the robot so that the robot will call his name. This behavior was frequently observed during the field trial. Finally, participants returned RFID tags at the exit desk near the entrance and completed a questionnaire about their impressions of the robots (Details were explained in [12], a field survey about impressions and anxieties).

\section{B. RFID tags and readers}

Active-type RFID tags and readers were used (Figure 4). We installed 18 RFID readers in the floor (Figure 5). RFID tags were embedded in a nameplate (5 $\mathrm{cm}$ in diameter) so that participants were easy to carry. Each RFID tag periodically transmitted its ID, which was received by each of the readers. The reader has eight degrees of attenuation (a mechanism that increases electrical resistance to weaken received radio signals) that reduces the maximum gain of the receiver by $12.5 \%$ with each step. This enables us to estimate the rough distance between tags and readers. Detection areas were affected by the antenna positions of the RFID readers and signal reflections from walls. We measured the detection area of each RFID reader to adjust the distance parameters for each attenuation parameter. Each RFID 
reader changed attenuation parameters from five to eight at 0.75 second intervals. In addition, it costs 0.25 seconds to stabilize detection in each range. Thus, each reader can observe the distance of tags every 4 seconds.

\section{Position estimation with multiple RFID readers}

We integrated the output from all 20 RFID readers (two attached to the robot and 18 installed to the environment) to achieve approximate position estimation of each RFID tag. Figure 6 shows the outline of the estimation method, which is based on the Markov chain Monte Carlo method [13]. It projects the probability of the tag position to a map image of the floor. The size of the map image is 1024 x 512 pixels; thus, the area of each pixel represents $8 \mathrm{~cm}^{2}$. The system calculates the probability of each tag existence in each pixel by the detected signal strength of each RFID reader. The probability of tag existence is distributed equally to areas ( $\mathrm{x}$, y) that satisfy the following two equations:

$$
\begin{aligned}
& \left(x-c x_{A}\right)^{2}+\left(y-c y_{A}\right)^{2}>r_{A}\left(N_{A}^{a}-1\right)^{2}, \\
& \left(x-c x_{A}\right)^{2}+\left(y-c y_{A}\right)^{2}<r_{A}\left(N_{A}^{a}\right)^{2},
\end{aligned}
$$

where $\left(c x_{A}, c y_{A}\right)$ denote the position of RFID reader ' $A$ ' in the $\mathrm{X}-\mathrm{Y}$ coordinate. $r_{A}(n)$ denotes the pre-measured radius for RFID reader ' $A$ ' within which it can detect RFID tags with attenuation parameter $n . N_{A}^{a}$ is signal strength for RFID tag 'a' (minimum attenuation parameter within which 'A' could detect ' $a$ '). The system adds the probabilities of tag existence when areas overlap and normalizes the probability of each tag's existence calculated by each RFID reader.

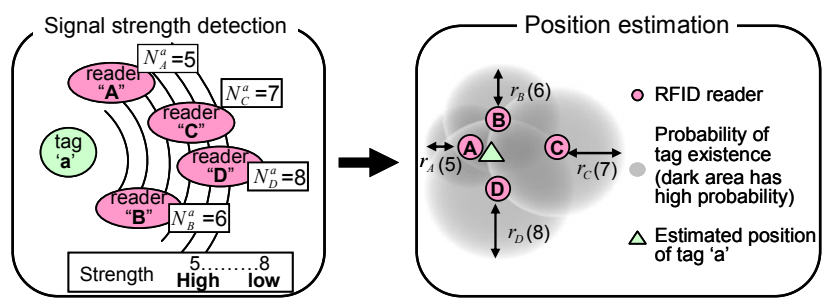

Figure 6: Outline of position estimation method

To decrease observation noise, it incorporates the previous position into the estimation of the next tag position in the following process (This is a standard process of the Markov chain Monte Carlo method [13]):

(1) Acquire weighted sample set $\left\{\left(s_{t-1}^{(n)}, \pi_{t-1}^{(n)}\right), n=1, \ldots, N\right\}$ at time-step $t$ - 1 from a previous iteration. $s_{t-1}^{(n)}$ and $\pi_{t-1}^{(n)}$ denote the $n$-th sampling point (X-Y coordination) and its weight at $t-1$ (that is, the probability of tag existence at position $s_{t-1}^{(n)}$ ).

(2) Calculate cumulative weights $c_{t-1}^{(n)}$ as

$c_{t-1}^{(0)}=0$, and $c_{t-1}^{(n)}=c_{t-1}^{(n-1)}+\pi_{t-1}^{(n)}$,

and select sample set $\left\{s_{t-1}^{\prime(n)}\right\}$ by iterating the following steps:

(a) Generate a random number $r \in[0,1]$, uniformly distributed.

(b) By binary subdivision, find the smallest $j$ for which $c_{t-1}^{(j)} \geq r$.

(c) Set $s^{\prime(n)}=s_{t-1}^{(j)}$.

(3) Measure observation features at new sample position $s_{t}^{(n)}$, and calculate weight $\pi_{t}^{(n)}$ using equations (1) and (2).

We evaluated the accuracy of estimated positions with this method. Several people walked around the floor simulating typical visiting behavior of museum visitors. We compared their real trajectories and estimated trajectories. The error in the estimated positions was approximately $2.8 \mathrm{~m}$. We believe that there were several sources of errors including the reflection of and instability of radio signals and the moving of RFID tags due to the movement of subjects.

\section{ANALYSIS OF TRAJECTORIES}

We gathered 8,091 visitor trajectories during the 25-day field trial ${ }^{1}$. Since 49,091 people visited the science museum, approximately $16.5 \%$ participated in the field trial. Participant IDs, trajectories (sequence of positions), and questionnaire answers were stored in a database in real-time.

Here, we define the basic symbols used in the paper. Since observations from each RFID reader are asynchronous, we need to synchronize every visitor position. $\boldsymbol{P}_{\boldsymbol{t}_{\boldsymbol{i}}}^{\boldsymbol{i}}$ denotes the positions of visitors in the X-Y coordinates, where $i$ denotes visitor ID and $t_{i}$ denotes observed time. The position data for person $i$ is updated every time one RFID reader detects the tag of person $i$; thus, the duration between $t_{i}$ and $t_{i+1}$ is not constant. It mostly ranged within 0 to several seconds. The position of visitor $i$ at time $t$ is linear interpolation, calculated as:

$$
\boldsymbol{P}^{\boldsymbol{i}}(\boldsymbol{t})=\alpha \boldsymbol{P}_{t_{i}}^{\boldsymbol{i}}+(1-\alpha) \boldsymbol{P}_{t_{i+1}}^{\boldsymbol{i}},
$$

where $\alpha=\left(\boldsymbol{t}-\boldsymbol{t}_{\boldsymbol{i}}\right) /\left(\boldsymbol{t}_{\boldsymbol{i}+1}-\boldsymbol{t}_{\boldsymbol{i}}\right)$ and $\boldsymbol{t}_{\boldsymbol{i}}<\mathrm{t}<\boldsymbol{t}_{\boldsymbol{i}+1}$. (If the duration between $\boldsymbol{t}_{\boldsymbol{i}}$ and $\boldsymbol{t}_{\boldsymbol{i}+1}$ is larger than 30 seconds, we treated $\boldsymbol{P}^{i}(\boldsymbol{t})$ as a missing data point.)

The trajectories of visitors $\boldsymbol{P}^{i}$ are defined as:

$$
\boldsymbol{P}^{i}=\left(\boldsymbol{P}^{i}\left(\boldsymbol{t}_{i}\right), \boldsymbol{P}^{i}\left(\boldsymbol{t}_{i}+\Delta t\right), \boldsymbol{P}^{i}\left(t_{i}+2 \cdot \Delta t\right), \cdots, \boldsymbol{P}^{i}\left(t_{f}\right)\right),
$$

where $t_{i}$ and $t_{f}$ denote the start and end time of observations. We set $\Delta t$ as 4 seconds based on the detection frequency of an RFID reader. We refer to $\boldsymbol{P}^{i}$ as a "trajectory" hereinafter.

\section{A. Space \\ 1) Use of space}

We analyzed all 8,091 trajectories to identify how the space was used. Figure 7 shows the distribution of positions where participants remained. Red (darker in grayscale image) represents positions where visitors remained for amounts of time of more than average plus $2 \sigma$ (standard deviation). If the amount equals the average, the software colors the position pink. If less than average minus $2 \sigma$, it colors it white. Two green triangles and 18 green circles represent the locations of RFID readers. The following findings were produced regarding visitor movement in the museum:

- The upper left area was crowded because this is where the robots and "child-friendly" exhibits were placed, such as

\footnotetext{
${ }^{1}$ The trial was actually performed for two months, but since we changed locations of the RFID readers after one month, we only used the data from the second month (Aug. 7 to Aug. 31, 2003).
} 


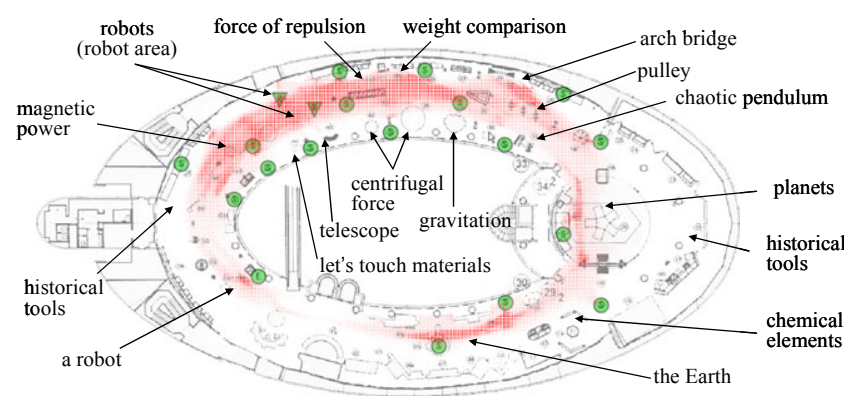

Figure 7: Distribution of positions where participants lingered

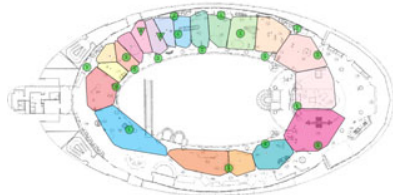

(a) $\mathrm{k}=20$

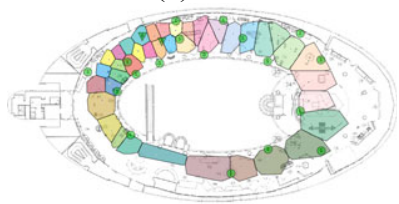

(c) $\mathrm{k}=40$

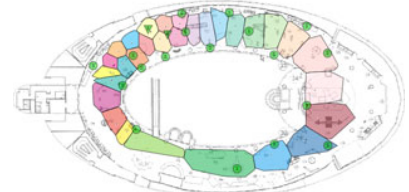

(b) $\mathrm{k}=30$

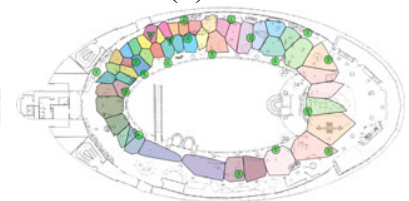

(d) $\mathrm{k}=50$
Figure 8: Spatial partitioning with different $k$ values

"let's touch materials" and "magnetic power" activities.

- In the upper right, the region around "pulley" and "arch bridge" activities was crowded while the area around "chaotic pendulum" was not as crowded.

- The right region around "planets" and "historical tools" activities was not crowded.

However, two limitations must be considered in the context of these findings:

- Positions in the areas that were not surrounded with RFID readers were inaccurate (particularly, in the "planets" area).

- Our method did not accurately estimate positions if participants are positioned upon exhibits because some exhibits are partly made of iron, such as "centrifugal force" and "gravitation," which offered people rides to experience the force.

\section{2) Spatial partitioning}

To analyze the use of space, we conducted spatial partitioning, which was also used in the following trajectory analysis to reduce calculation cost. A k-means algorithm was applied to all trajectories to divide the museum into $k$ areas. Figure 8 and Table 1 show the results of spatial partitioning with different $k$ values. As Figure 8 shows, areas in which visitors remained for significant amounts of time, such as the robot area, are represented by small clusters. This reflects the center of balance of each cluster; a more crowded point is likely to be the center of a cluster, and an uncrowded point is likely to be the periphery of a cluster.

Regarding appropriate $k$ value, there is a tradeoff. If the number of clusters is large, we can gather more precise information about trajectories, but these results will be more influenced by errors of position estimation (error is about 2.8

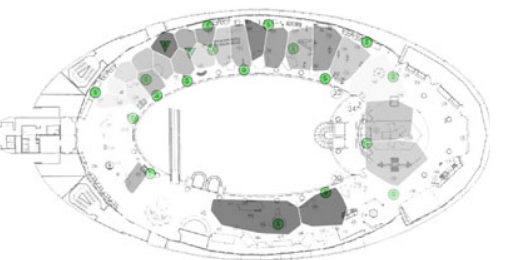
tioning
Figure 9: Distribution based on parti-

\begin{tabular}{|r|r|}
\hline num. of cluster & s.d. \\
\hline 10 & 2.94 \\
\hline 20 & 1.73 \\
\hline 30 & 1.56 \\
\hline 40 & 1.30 \\
\hline 50 & 1.14 \\
\hline 60 & 0.98 \\
\hline
\end{tabular}

Table 1: Various partitioning sizes m). This also affects visualization. As Figure 8 shows, a larger $k$ value complicates the interpretation of results. Considering these issues, we chose a $k$ value of 30 .

Figure 9 shows the distribution of spaces based on spatial partitioning in relation to the amount of time participants remained. Our software assisted visualization by representing crowded areas with darker colors. If the number of points $\left(P^{i}(t)\right)$ included in the partition equals the average, the software colors the area middle gray. If more than average plus $2 \sigma$, it colors it black. If less than average minus $2 \sigma$, it colors it white.

\section{B. Visiting patterns}

Based on the estimated trajectories, we analyzed how people visited the museum exhibits. In this section we introduce a method of extracting typical visiting patterns, which, as a result, helps us retrieve atypical visiting behavior.

1) Preparation: State chain models

We analyzed trajectories based on the state chain model illustrated in Figure 10. That is, we converted the trajectory based on X-Y coordination, $\boldsymbol{P}^{i}$, to a sequence of states, $\boldsymbol{S}^{i}$, based on spatial partitioning. It is defined as,

$$
\boldsymbol{S}^{\boldsymbol{i}}=\bigcup_{\boldsymbol{p} \in \boldsymbol{P}^{i}}\left\{\boldsymbol{s}_{\boldsymbol{t}}=\boldsymbol{n} \mid \boldsymbol{p} \in \boldsymbol{A}_{\boldsymbol{n}}\right\},
$$

where $A_{n}$ is the partition the point in trajectory $p$ belongs.

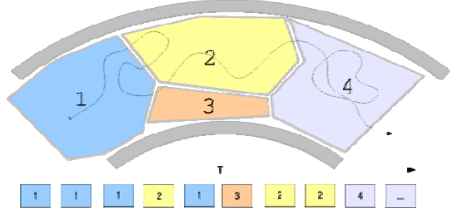

Figure 10: State chain model

We calculate the distance between two state chains, $\boldsymbol{S}^{i}$ and $\boldsymbol{S}^{j}$, by using a DP matching method, which is identical to the comparison of strings known as the Levenshtein distance. Figure 11 compares trajectory works. Here, we set the distance between partitions as the distance between the centers of the partitions. Thus, "insert" and "delete" operation costs partition distance plus a constant parameter, which represents the tradeoff cost between time and space.
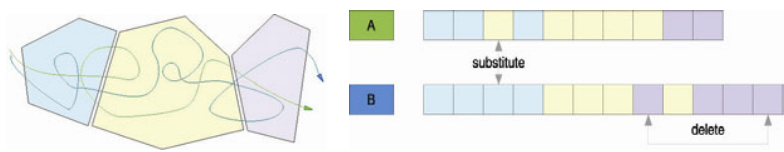

(a) two trajectories (b) comparison of state chains of trajectories

Figure 11: Comparison of trajectories based on DP matching 


\begin{tabular}{|r|r|r|}
\hline \multicolumn{1}{|l|}{$\mathrm{k}$} & \multicolumn{1}{|c|}{ S.D. } & calc. costs \\
\hline 2 & 20.9 & 40903.7 \\
\hline 3 & 18.2 & 17144.3 \\
\hline 4 & 17.5 & 10362.9 \\
\hline 5 & 14.6 & 7278.8 \\
\hline 6 & 14.7 & 7208.0 \\
\hline 7 & 12.8 & 9493.3 \\
\hline 8 & 14.1 & 8789.4 \\
\hline 9 & 12.4 & 6449.9 \\
\hline 10 & 12.2 & 6691.7 \\
\hline
\end{tabular}

Table 2: Standard deviation and calculation costs [sec.]

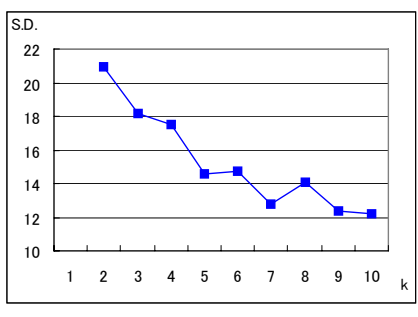

Figure 12: Illustration of standard deviations

This state-chain representation reduces calculation cost For example, we compared calculation cost based on raw trajectory $\boldsymbol{P}^{i}$ and state chain $\boldsymbol{S}^{i}$ for retrieving typical visiting patterns (details explained in 3.B.3) with a k-means clustering method from 28 trajectories. The state-chain method costs $0.53 \mathrm{sec}$ while the raw-trajectory-based method costs $9.56 \mathrm{sec}$. Thus, using the state chain is eighteen times faster. We cached the calculation of distance between partitions in the state-chain-based method (that is, insert, delete, and substitute costs in DP matching), which greatly improved the calculation speed. Moreover, state chain is used in the visualization of visiting patterns, which is also its advantage. We will demonstrate it in the following section.

\section{2) Identification of typical visiting patterns}

We classified trajectories with a k-means method to identify typical visiting patterns. Although 8,091 trajectories were obtained, some were corrupted due to observation noise and error. Occasionally, the system failed to estimate the position of people, which resulted in missing points in the trajectory. We extracted 5,102 trajectories longer than 5 minutes whose missing duration is less than $10 \%$.

Predicated on the trajectory distance based on the state-chain comparison, we clustered these state chains with the k-means method. Table 2 and Figure 12 show the relationship between the number of clusters and standard deviations. Calculation times are done with a $3 \mathrm{GHz}$ Pentium IV computer. Since standard deviation largely dropped until $k=5$, we interpreted visiting patterns at $k=5$. Of course, we can analyze behavior patterns at any $k$ value; a larger number $k$ will result in more detailed separation of visiting patterns.

Figure 13 shows the visualized output of the global trends of each visiting pattern at $k=5$. In the figure, the frequency of remaining in a particular location is represented as color and transitions between adjacent areas as arrows. If the frequency is more than average plus $2 \sigma$, it is colored black. If less than average minus $2 \sigma$, it is colored white. The arrow from partition $i$ to $j$ indicates that $\left(N_{i j}-N_{j i}\right)$ is larger than a threshold (here, set as 0.2 ) where $N_{i j}$ indicates a transition from $i$ to $j$. If $\left(N_{i j}-N_{j i}\right)$ is near the threshold, the software outputs it as blue; and if around the threshold plus 2.0, the software indicates this using red (brighter color in grayscale). As shown in Figure 13, the following five typical patterns are retrieved at $k=5$ : (See Figure 7 for information of exhibit locations.)

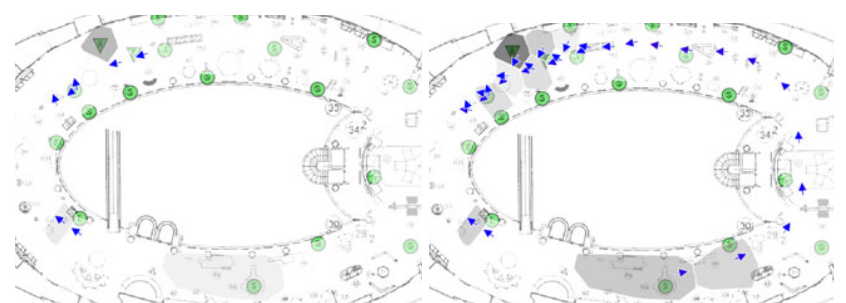

(1) Directly going to robot area (2) Going around and staying at robot area

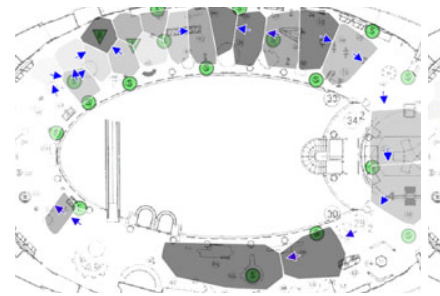

(3) Went around backwards

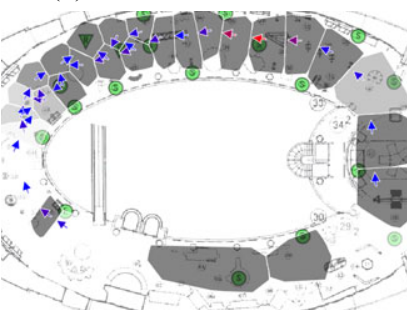

(5) Stayed for long time Distribution of visiting patterns Figure 13: Five typical behavior patterns of visitors

(1) Directly going to robot area: 346 people

People directly went to the robot area (green triangles displayed in the figure around the slightly crowded area) from the reception desk clockwise and interacted with the robots for a short time. After that, they went to the exit desk. In the figure, no arrows and no colored areas appeared around the bottom right and upper right areas, which represents that they did not go there.

(2) Going around and staying in the robot area: 1024 people People moved counter clockwise, which is the suggested direction from the entrance; but they rarely stayed at the exhibits and only stayed around the robot area. This caused the visualization output of arrows in the upper right.

(3) Went around backwards: 728 people

People directly went to the robot area from the reception desk, which resembles pattern 1. After that, they looked around the exhibits in a counter clockwise fashion.

(4) Visited at every place: 2726 people

People almost stayed at every place, particularly at "the Earth" exhibit; some stayed at the exhibits in the upper right area and the robot area.

(5) Stayed for long time: 278 people

Similar to pattern 4, but these people stayed in the floor for a long time (2488 to 7059 seconds) lingering at many exhibits. The relationships among the five patterns are also illustrated in the bottom right of Figure 13. It represents the similarity of participant trajectories by using a spring model, which is 
based on the distance between each trajectory as a spring force. Due to the heavy calculation of the spring model, we only used $10 \%$ of the trajectories. We can see the relationships among the visiting patterns. For example, pattern 2 and 4 are connected together while pattern 1 and 5 are placed in the both ends: which means they are very different.

\section{3) Identification of non-typical visiting behaviors}

Based on trajectory distance from the closest cluster center, we retrieved atypical visiting behavior, as shown in Figure 14. Figure 14(a) is one notable atypical visiting behavior, whose distance to the closest cluster center was 34.0. As shown in Table 2, the standard deviation of distance from the closest cluster center was 14.6 at $k=5$; therefore, 34.0 is a large value. This person left the field trial by returning his tag to a staff member who immediately performed his exit process at the robot area. As a result, his trajectory "disappeared." In the cases of (b) and (c), the participants only stayed around the entrance, which is unusual as people usually tried to interact with the robots. In the case of (d), the person stayed around the floor for $6776 \mathrm{sec}$ while the average length of stay was $1990 \mathrm{sec}$.
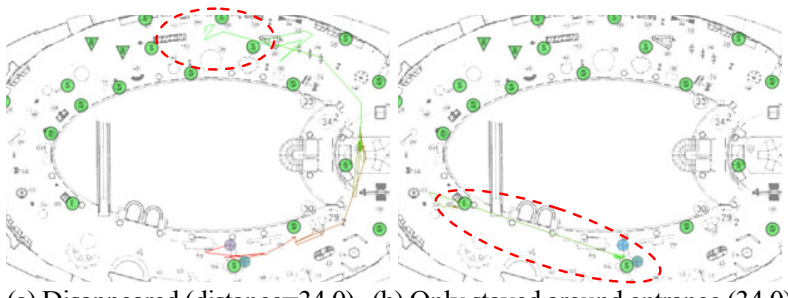

(a) Disappeared (distance $=34.0$ )

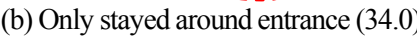

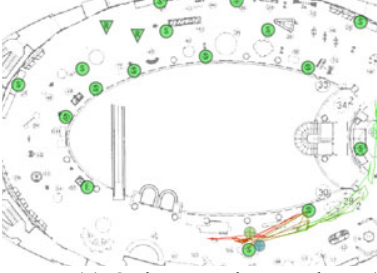

(c) Only stayed around

"the Earth" and "planets" (29.8)

(d) Started to visit in counter clockwise

" and stayed for long time (23.7) Figure 14: Examples of non-typical visiting behaviors (Path materialized by a ranging from red (darker color) to green (lighter).)

\section{4) Analysis of attributions}

We used a visualization technique to analyze how people's interests and attributions affected their visiting behavior. Figure 15 shows the results for attributions with respect to the robots. At the exit desk, some participants filled out questionnaires that asked about their interest in the robots on 1-to-5 scale [12]. Figure 15 on the left is the visiting pattern of people who answered low interest, and Figure 15 on the right is the visiting pattern of people who answered high interest. As the figure shows, the difference was very small.

Figure 16 illustrates the museum's crowdedness as an environmental factor affecting visiting patterns. From registration information, we calculated the "crowdedness" of each visitor's environment, based on the average number of registered people during their stay. For example, if there are 10 other registered visitors during a registered person's stay, the crowdedness for the person is calculated as 10.0. We compared upper quartile ("crowdedness" of more than 78.6, 1275 visitors) and lower quartile (lower than 20.4, 1275 visitors), but the difference was also small.

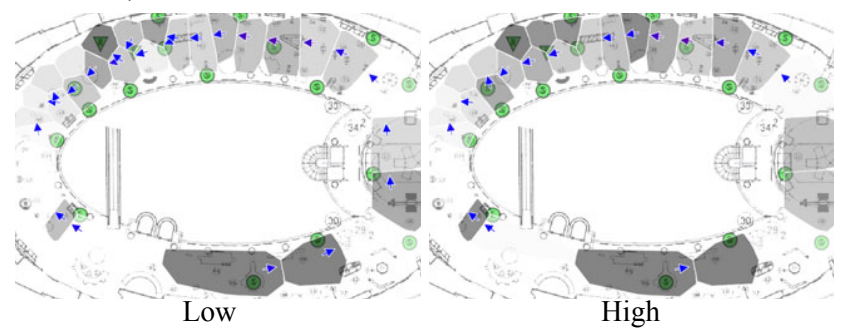

Figure 15: Interest in robots

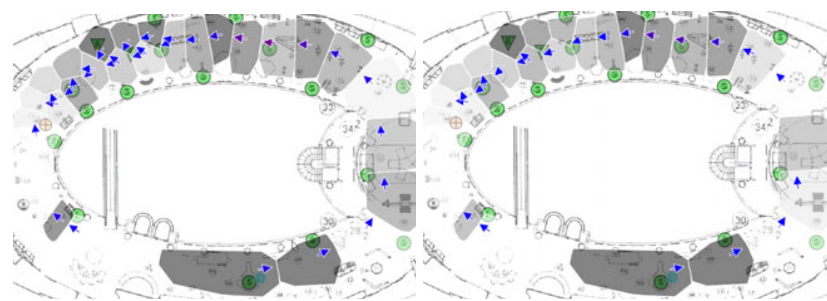

Low: less than 20.4 visitors High: more than 78.6 visitors

Figure 16: Crowdedness of environment

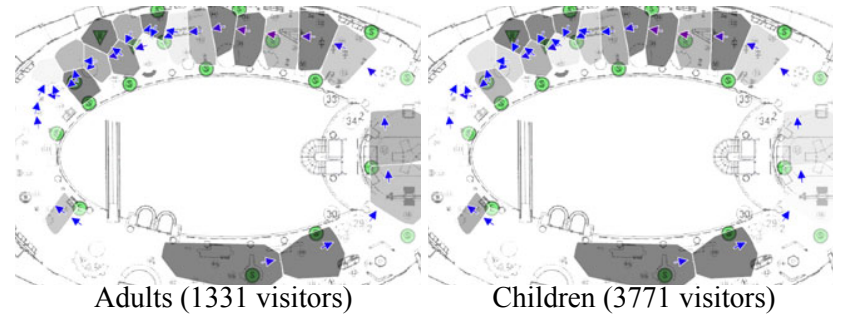

Figure 17: Adult-child attribution

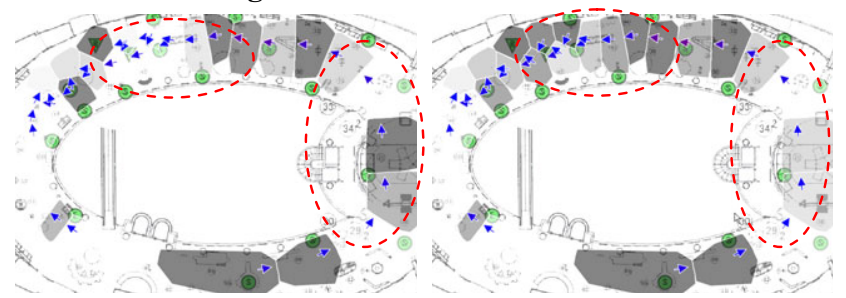

Without a child in groups (201 visitors) With a child in groups (4901 visitors)

Figure 18: Whether accompanying a child

We also analyzed participant attributions; that is, whether a participant is an adult or a child. Figure 17 shows the results of adult-child comparisons, which revealed a slight difference of staying time around the robot and planet areas. Moreover, we only retrieved people who came in with an adult. Figure 18 shows the comparison of "adults without a child in the group" and "people in the group including a child." It shows a large difference in the visiting of the robot and planets areas. A comparison of the left figure with the right shows a smaller staying time around the robot area and larger staying time around the planet area when without 


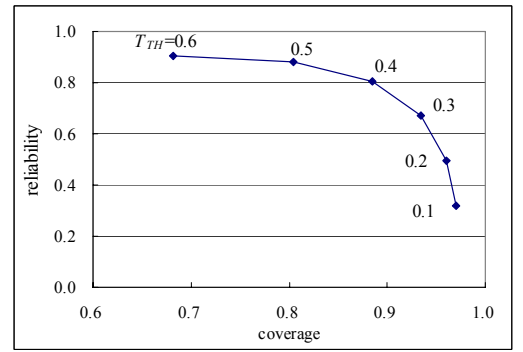

Figure 19: Estimation of group relationship

(Numbers besides the line represents threshold $T_{T H}$. For example, at $T_{T H}$ $=0.4$, it estimated $89 \%$ of group relationships with $81 \%$ reliability)

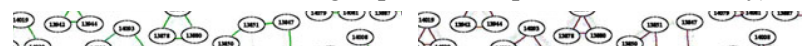

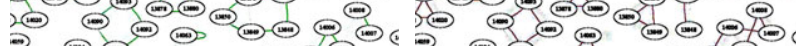

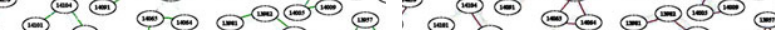

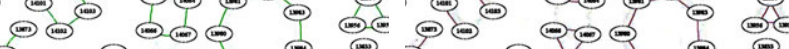

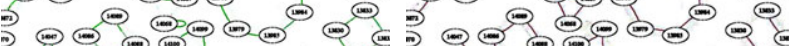

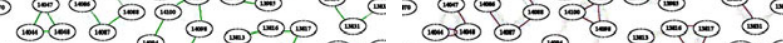

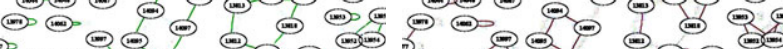

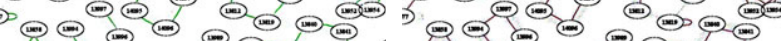
(.) (ब)

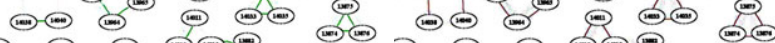

Figure 20: Example of estimated (left) and real (right) group relationship: circles represent a person, and links between persons represents a relationship

children (left figure) than with children (right figure). Thus, it suggests that visitor behavior largely depended on the presence of children who usually enjoyed interaction with the robots very much.

\section{Relationships}

A previous work demonstrates a robot's capability of estimating children's friendship based on measuring a period of simultaneous stay between two children [14]. Similarly, we estimated the friendliness of people based on co-visiting behavior in the science museum. Since visitors registered their names with the group members (we limited registration forms to one per group), we define members of a group as the visitors on the same registration form. We defined the estimated relationship between persons $A$ and $B(\operatorname{Group}(A, B))$ as:

$\operatorname{Group}(A, B)=$ if $\left(T_{A B} / \min \left(T_{A}, T_{B}\right)>T_{T H}\right)$,

$T_{A}=\sum$ if $($ obs $(A)) \cdot \Delta \mathrm{t}$,

$T_{A B}=\sum$ if $\left(o b s(A)\right.$ and $o b s(B)$ and $\left.\operatorname{dist}(A, B)<D_{T H}\right) \cdot \Delta \mathrm{t}$, where $o b s(A)$ becomes true only when any RFID reader observes the ID of person $A$, dist $(A, B)$ is calculated as $\left|\boldsymbol{P}^{\boldsymbol{A}}(\boldsymbol{t})-\boldsymbol{P}^{\boldsymbol{B}}(\boldsymbol{t})\right|$ at time $t$, and if () becomes 1 when the logical equation inside the parentheses is true (otherwise 0 ). We prepared two thresholds $T_{T H}$ and $D_{T H}$; threshold $T_{T H}$ is for the ratio of simultaneous interaction time, and threshold $D_{T H}$ is for the distance which the system uses to assume $A$ and $B$ are together. After this process, a graph-based refine of estimation was conducted to maintain the consistency of group relationships. In our trial, we set $\Delta t$ to four seconds (observation frequency of an RFID reader) and $D_{T H}$ to five meters.

Figure 19 indicates the estimation results with various parameters $\left(T_{T H}\right)$. Since the number of group relationships among all visitors was fairly small, we focused on the appropriateness (coverage and reliability) of the estimated relationships instead of the rate of correct classification (precision and recall in web search techniques), which are defined as follows:

$$
\begin{aligned}
& \text { Coverage }=\frac{\text { correct relationships in estimated relationships }}{\text { all correct relationships }} \\
& \text { Reliability }=\frac{\text { correct relationships in estimated relationships }}{\text { estimated relationships }}
\end{aligned}
$$

Here, correct relationships denotes the group relationships gathered from registration forms, and estimated relationships are given by our estimation method. The line in the figure has several points corresponding to different $T_{T H}$. There is obviously a tradeoff between reliability and coverage that is controlled by $T_{T H}$. As a result, for example, our method estimated about $68 \%$ coverage of the relationships with $91 \%$ reliability (at $T_{T H}=0.6$ ) and $89 \%$ coverage with $81 \%$ reliability (at $T_{T H}=0.4$ ). In other words, this result also indicates that in the museum, $89 \%$ of visitors spent $40 \%$ of their time with their group members. Figure 20 shows an example of estimated relationships and real relationships.

\section{DisCUSSIONS AND CONCLUSION}

\section{A. Summary of findings}

This paper revealed the extent to which we can obtain information from people's trajectories in a science museum using RFID tags. We found the following:

1. We obtained 5,102 clean trajectories of visitors within 2.8 $\mathrm{m}$ distance error using 20 RFID readers placed in the $84 \mathrm{x}$ $42 \mathrm{~m}$ environment during the 25 -day field trial.

2. The trajectories enable us to see how the space was used, such as which areas were crowded and uncrowded. For example, "the Earth" and robot areas were crowded while the "planets" area was not so crowded.

3. The trajectories enabled us to recognize typical visiting patterns. For example, people in "visited at every place" pattern lingered around "the Earth" exhibit for a while, quickly passed the "planets" exhibit area, stayed around the "pulley" area, and then interacted with the robots.

4. The trajectories enabled us to identify participants with atypical behavior. For example, it detected a person who only stayed around "the Earth" exhibit near the entrance.

5. We revealed to what extent people's trajectories are related to their subjective impressions and attributions. There is a stronger correlation between trajectories and attributions, particularly whether the group consists of only adults.

6. We estimated group membership based on trajectories.

We believe that these demonstrate the effectiveness of the method in inferring complex visiting behavior in the museum.

\section{B. Contribution to the method of analyzing trajectories}

This study demonstrated one successful method of ana- 
lyzing people's trajectories obtained by ubiquitous sensors and an RFID tag system. The method consists of analysis function and visualization support function. Regarding the analysis, it converts trajectories to state chains based on partitions, calculates similarities between two trajectories based on the DP matching method, and makes clusters of thousands of trajectories with the k-means method. It also accurately estimates group-membership between visitors by observing co-visiting behavior. Regarding the visualization support, the software displays staying times and transitions based on partitions, our software enabled us to visualize the following: trajectories, how the space was used, typical and atypical visiting patterns, and the relationships between trajectories and attributions.

To explore optimal algorithm will be our future work. Our method is combination of available straight-forward methods. The k-means clustering with DP matching worked well but its calculation cost is heavy. Several methods analyze time-sequence data; however, note that an appropriate algorithm also depends on the quality of position estimation. In our case, position estimation based on RFID resulted in $2.8 \mathrm{~m}$ error. Although we tried to apply a method based on Bayesian clustering with a Markov-chain model, it failed due to the noisiness of state transitions.

\section{Implication for network robot system}

The developed method is a powerful tool for developing network robot system (or, ubiquitous robotics), since it enables to recognize capability of visitors' activity (visiting pattern) and relationship, particularly in a museum environment. For example, since this method enables us to classify people's visiting patterns, we will be able to efficiently prepare the interactive behavior of robots. The robot may talk more about robotics to the people in the "directly going to the robot" visiting pattern, and it may talk more about the science museum to people in the "stayed for long time" visiting pattern. Identifying atypical visiting behavior can also contribute to optimizing robot behavior, because the person whose behavior is atypical may need special services. The estimation of group relationships can also contribute to promoting human-robot interaction. For example, the robot may promote conversation among visitors who are within estimated group relationship by talking such as "Are you with friends today?" In other words, we can prepare several types of robot behavior according to the detected visiting patterns, but utilizing estimated information about people's behavior and relationships is one future research area.

\section{D.Privacy issues}

Privacy is a concern that may arise from this study. As a scientific investigation, we conducted this study to identify what we can estimate from people's trajectories. It was never our intention to force "every" visitor to wear RFID tags and make their private experience public. In this study, we in- formed participants that the recorded and obtained information would be carefully managed and only used for research purposes. We only provided RFID tags to visitors who completed a consent form. When a system that employs this technology is used, people must have a choice to participate, which balances privacy concerns and benefits from system.

\section{E. Limitations}

This study was conducted in a science museum where humanoid robots were exhibited. Thus, limitations exist about the degree to which we can generalize its findings. We, however, expect to find similar trends in other exhibition environments, since the presence of specific robots can probably be generalized as other popular exhibits.

\section{ACKNOWLEDGMENT}

We wish to thank the staff at the Osaka Science Museum for their highly appreciated cooperation. We also with to thank people at ATR for their cooperative support: H. Terauchi, T. Shibata, K. Hayashi, M. Kakio, T. Tajika, F. Yamaoka, D. Eaton, T. Tasaki, K. Sugahara, and B. Noorani.

\section{REFERENCES}

[1] T. Kanda, T. Hirano, D. Eaton, and H. Ishiguro, Interactive Robots as Social Partners and Peer Tutors for Children: A Field Trial, Human Computer Interaction, Vol. 19, No. 1-2, pp. 61-84, 2004.

[2] M. Shiomi, T. Kanda, H. Ishiguro, and N. Hagita, Interactive Humanoid Robots for a Science Museum, ACM 1st Annual Conference on Human-Robot Interaction (HRI2006), 2006.

[3] J. Letchner, D. Fox, and A. LaMarce, Large-Scale Localization from Wireless Signal Strength, Proc. of the National Conference on Artificial Intelligence (AAAI-05), 2005.

[4] D. Schulz, D. Fox, and J. Hightower, People Tracking with Anonymous and ID-sensors Using Rao-Blackwellised Particle Filters, Int. Joint Conf. on Artificial Intelligence (IJCAI-03), 2003.

[5] A. Madhavapeddy, and A. Tse, A study of Bluetooth propagation using accurate indoor location mapping, Int. Conf. Ubiquitous Computing (Ubicomp2005), pp. 105-122, 2005.

[6] H. Yan and M. J. Mataric, General Spatial Features for Analysis of Multi-robot and Human Activities from Raw Position Data, IEEE/RSJ Int. Conf. on Robotics and Intelligent Systems (IROS02), pp 2770-2775, 2002

[7] N. Eagle, and A. Pentland, Reality Mining: Sensing Complex Social Systems, Personal and Ubiquitous Computing, online first, Nov., 2005.

[8] L. Liao, D. Fox, and H. Kautz, Location-Based Activity Recognition using Relational Markov Networks, Int. Joint Conf. on Artificial Intelligence (IJCAI-05), 2005.

[9] T. Choudhury and A. Pentland, Modeling Face-to-Face Communication using the Sociometer, Int. Conf. Ubiquitous Computing, 2003

[10] F. Sparacino, The Museum Wearable, Proc. of Museums and the Web, 2002.

[11]B. Serrell, The question of visitor styles. Visitor Studies: Theory, Research, and Practice 7(1): 48-53, 1996

[12]T. Nomura, et al, Questionnaire-Based Research on Opinions of Visitors for Communication Robots at an Exhibition in Japan, Int. Conf. on Human-Computer Interaction (Interact 2005), 2005.

[13] M. Isard, and A. Blake, CONDENSATION - Conditional Density Propagation for Visual Tracking, Int. Journal of Computer Vision, Vol. 29, 1998.

[14]T. Kanda, and H. Ishiguro, Reading human relationships from their interaction with an interactive humanoid robot, Int. Conf. on Industrial and Engineering Applications of Artificial Intelligence and Expert Systems (IEA/AIE), pp. 402-412, 2004. 